\title{
Connecting Dense Gas Tracers of Star Formation in our Galaxy to High-z Star Formation
}

\author{
Jingwen $\mathrm{Wu}^{1}$, Neal J. Evans $\mathrm{II}^{1}$, Yu Gao ${ }^{2}$, Philip M. Solomon ${ }^{3}$, Yancy L. Shirley ${ }^{4,5}$, Paul \\ A. Vanden Bout ${ }^{6}$
}

\begin{abstract}
Observations have revealed prodigious amounts of star formation in starburst galaxies as traced by dust and molecular emission, even at large redshifts. Recent work shows that for both nearby spiral galaxies and distant starbursts, the global star formation rate, as indicated by the infrared luminosity, has a tight and almost linear correlation with the amount of dense gas as traced by the luminosity of HCN. Our surveys of Galactic dense cores in HCN 1-0 emission show that this correlation continues to a much smaller scale, with nearly the same ratio of infrared luminosity to HCN luminosity found over 7-8 orders of magnitude in $L_{I R}$, with a lower cutoff around $10^{4.5} \mathrm{~L}_{\odot}$ of infrared luminosity. The linear correlation suggests that we may understand distant star formation in terms of the known properties of local star-forming regions. Both the correlation and the luminosity cutoff can be explained if the basic unit of star formation in galaxies is a dense core, similar to those studied in our Galaxy.
\end{abstract}

Subject headings: stars: formation — ISM: molecules

\footnotetext{
${ }^{1}$ Department of Astronomy, The University of Texas at Austin, 1 University Station, C1400, Austin, Texas 78712-0259, jingwen@astro.as.utexas.edu, nje@astro.as.utexas.edu

${ }^{2}$ Purple Mountain Observatory, Chinese Academy of Sciences, 2 West Beijing Road, Nanjing 210008. P.R.China, yugao@pmo.ac.cn

${ }^{3}$ Department of Physics and Astronomy, SUNY at Stony Brook, Stony Brook, NY 11974, Philip.Solomon@sunysb.edu

${ }^{4}$ Steward Observatory, University of Arizona, Tucson, AZ 85721. yshirley@as.arizona.edu

${ }^{5}$ Bart J. Bok Fellow.

${ }^{6}$ National Radio Astronomy Observatory, Socorro, NM 87801, pvandenb@nrao.edu
} 


\section{Introduction}

Recent work has revealed large amounts of dust and molecules in starburst galaxies, even at large redshifts (e.g. Isaak et al. 2002; Reuland et al. 2003; Greve et al. 2005; Solomon \& Vanden Bout 2005). Understanding star formation in galaxies at high redshift is a critical step in understanding the formation of galaxies in the early Universe.

The simplest and most widely used relations between the star formation rate and a property of the interstellar medium (ISM) are the so-called "Schmidt laws". Schmidt (1959) proposed that the star formation rate was proportional to $\rho^{2}$, where $\rho$ is the gas volume density. In their modern form, these "laws" relate the surface density of star formation to the surface density of gas:

$$
\Sigma_{S F R}=A \Sigma_{\text {gas }}^{N}
$$

(e.g., Kennicutt 1998). The index $N$ has been inferred by various authors to be in the range of 1 to 2 (Kennicutt 1997). Measurements of HI, CO, and $\mathrm{H} \alpha$ on a large sample of normal spiral galaxies and starburst galaxies (Kennicutt 1998) could be fitted with $N=1.4$. It should be noted that for many of these galaxies, particularly the starburst galaxies, in the Kennicutt sample the surface density or surface brightness was not measured but obtained only by dividing the luminosity by a characteristic size often obtained from another parameter.

On a global scale, including luminous and ultraluminous starburst galaxies, there is a correlation between the total luminosity of far-infrared emission, which traces the star formation rate (e.g. Sanders and Mirabel 1996; Kewley et al. 2002), and the total luminosity of CO, tracing the molecular gas mass. However, this relation is not linear; the ratio of $L_{I R}$ to $L_{C O}$ increases with increasing $L_{I R}$ ( Sanders and Mirabel 1996; Solomon et al. 1997; Gao \& Solomon 2004a, 2004b; Solomon \& Vanden Bout 2005). Does this variation reflect an increase in "efficiency" of star formation, or an increasing failure of CO to trace the gas that is relevant to star formation?

The latter possibility is suggested by data on $\mathrm{HCN} J=1-0$ in galaxies. A recent survey of HCN $J=1-0$ emission in 65 normal spiral and starburst galaxies found that the star formation rate, as traced by the infrared luminosity, has a tight and linear correlation with the luminosity of HCN (Gao \& Solomon 2004a, 2004b). Those authors argued that CO is not linearly correlated with star formation because it traces only the low density GMC envelopes, not the really active star-forming part, the dense cores. The critical molecular parameter that measures star formation rates in galaxies is the amount of dense molecular gas, measured by the HCN luminosity. Because HCN traces the dense gas better than CO, $\mathrm{L}_{H C N}$ has a tighter correlation with $L_{I R}$ than does $L_{C O}$. The correlation remains linear over a factor of $10^{3}$ in luminosity for both normal galaxies and extreme starbursts, like luminous 
and ultraluminous infrared galaxies (LIRGs and ULIRGs, see Sanders and Mirabel, 1996). Gao and Solomon (2004a) therefore argue that both normal galaxies and starbursts should have the same star formation rate per amount of dense gas.

At lower luminosities, the relation for galaxies between $L_{I R}$ and $L_{C O}$ is linear, but the ratio between $L_{I R}$ and $L_{C O}$ has a dispersion of an order of magnitude. An even larger variation in $L_{I R} / L_{C O}$ (several orders of magnitude) is seen in Galactic clouds (Mooney \& Solomon 1988; Evans 1991; Mead et al. 1990). The dispersion in the relation for galaxies is less if $L_{H C N}$ is used instead of $\mathrm{CO}$; the same is true for other tracers of dense gas in Galactic cores (e.g. Mueller et al. 2002, Shirley et al. 2003), suggesting that studies of Galactic star formation can shed light on the trends in other galaxies.

In the Milky Way, star formation is dominated by clustered star formation (Carpenter 2000; Lada \& Lada 2003 ). Clustered star formation produces stars with a range of masses, but massive stars form nearly exclusively in clusters within massive dense cores. Since massive stars dominate the luminosity, they are the stars directly probed in studying star formation in other galaxies. Thus, understanding the relation between star formation and massive, dense cores in our Galaxy may shed light on the starburst phenomenon.

These cores are dense, turbulent, and dusty. They are well identified by the continuum emission from dust (e.g., Mueller et al. 2002) and line emission from molecular dense gas tracers like CS (Plume et al. 1992, 1997; Shirley et al. 2003) and HCN (Wu \& Evans 2003). In our previous work on a large sample of massive cores, we found that the bolometric (almost all far-infrared) luminosity of the cores is roughly proportional to the mass inferred from the dust emission (Mueller et al. 2002) and the virial mass determined from CS (Shirley et al. 2003). This result suggests that a relation between $L_{I R}$ and $L_{H C N}$ may exist in Galactic dense cores, possibly similar to that in starburst galaxies.

One difficulty is that systematic studies of the Galactic dense cores have used dust continuum emission and CS, while HCN has been more commonly used for studies of galaxies. To facilitate comparison with the HCN $J=1-0$ galaxy survey, we have mapped the HCN $J=1-0$ transition in a sample of 47 Galactic star forming cores (Wu et al. 2005, in preparation). In this paper we will summarize the results from this survey in connection with the HCN surveys of other galaxies.

The sample mapped in HCN $J=1-0$ is mostly a subset of a larger sample of Galactic massive dense cores that have been mapped in CS and dust emission (Shirley et al. 2003; Mueller et al. 2002). The sources in this category have infrared luminosities ranging from $10^{3}-10^{7} \mathrm{~L}_{\odot}$ and most contain compact or ultracompact $\mathrm{H} \amalg(\mathrm{UCH} \amalg)$ regions. To extend the sample towards lower luminosities, we selected 14 IRAS sources from outflow surveys 
(Zhang et al. 2005; Wu et al. 2004) and a few from other publications.

\section{Observation \& Data Analysis}

Observations of HCN $J=1 \rightarrow 0(88.6318473 \mathrm{GHz})$ on Galactic dense cores were made with the 14-m telescope of the Five College Radio Astronomy Observatory (FCRAO) in 2004 April, December and 2005 February. The FWHM of the beam for this frequency is $58^{\prime \prime}$. The 16-element focal plane array (SEQUOIA) was used, with typical system temperatures 100200K. A velocity resolution of $0.1 \mathrm{~km} \mathrm{~s}^{-1}$ was achieved with the $25 \mathrm{MHz}$ bandwidth on the dual channel correlator (DCC). We convert the measured $T_{A}^{*}$ to $T_{R}$ via $T_{R}=T_{A}^{*} /\left(\eta_{F S S} \eta_{c}\right)$, with $\eta_{F S S}=0.7$. The value of $\eta_{c}$ depends on source size; for the typical map in this study $\left(\sim 10^{\prime}\right), \eta_{C}=0.7$. The map size was extended until the edge of the HCN $J=1 \rightarrow 0$ emission was reached, typically at the $2 \sigma$ level (mean $\sigma \sim 0.3 \mathrm{~K} \mathrm{~km} / \mathrm{s}$ ), so we could get the total HCN luminosity.

Maps of HCN 1-0 emission were obtained for 47 Galactic star-forming cores. Detailed results will be presented elsewhere (Wu et al. 2005, in preparation), but we give a few properties of the sample here, which are relevant to this paper. More than $90 \%$ of the cores were well resolved by the maps. We focus on these resolved sources in this paper.

The size of the core is characterized by the nominal core radius after beam deconvolution, $R_{H C N}$, the radius of a circle that has the same area as the half peak intensity contour:

$$
R_{H C N}=D\left(\frac{A_{1 / 2}}{\pi}-\frac{\theta_{\text {beam }}^{2}}{4}\right)^{1 / 2},
$$

where $A_{1 / 2}$ is the area within the contour of half peak intensity. The median $R_{H C N 1-0}$ of the sample is $0.48 \mathrm{pc}$.

The HCN line luminosity of each core, assuming a gaussian brightness distribution for the source and a gaussian beam, is:

$$
L_{H C N}=23.5 \times 10^{-6} \times D^{2} \times\left(\frac{\pi \times \theta_{s}^{2}}{4 \ln 2}\right) \times\left(\frac{\theta_{s}^{2}+\theta_{\text {beam }}^{2}}{\theta_{s}^{2}}\right) \times \int T_{R} d v
$$

Here $\mathrm{D}$ is the distance in the unit of $\mathrm{kpc}, \theta_{s}$ and $\theta_{\text {beam }}$ are the angular size of the source and beam in arcsecond. This method is parallel to that of Gao \& Solomon (2004b), but adapted to Galactic cores. $L_{H C N}$ ranges from 0.4 to $8000 \mathrm{~K} \mathrm{~km} \mathrm{~s}^{-1} \mathrm{pc}^{2}$, with the median value of 80 $\mathrm{K} \mathrm{km} \mathrm{s}{ }^{-1} \mathrm{pc}^{2}$.

The total infrared luminosity $(8-1000 \mu \mathrm{m})$ was calculated based on the 4 IRAS bands 
(Sanders and Mirabel 1996), as was done for the galaxy sample of Gao \& Solomon (2004a):

$$
L_{I R}=0.56 \times D^{2} \times\left(13.48 \times f_{12}+5.16 \times f_{25}+2.58 \times f_{60}+f_{100}\right),
$$

where $f_{x}$ is the flux in band $x$ from the four IRAS bands in the units of Jy, D in kpc, and $\mathrm{L}_{I R}$ in $\mathrm{L}_{\odot}$.

\section{Comparison of Milky Way and galactic Relations}

The derived $L_{I R}$ and $L_{H C N}$ are plotted in a log-log diagram in Fig. 1 to compare with data on galaxies from Gao \& Solomon (2004a). The correlation of $L_{I R}-L_{H C N}$ extends from galaxy scales to the much smaller scales of Galactic molecular cores. The fit for Gao \& Solomon's galaxy sample is $\log \left(L_{I R}\right)=1.00 \times \log \left(L_{H C N}\right)+2.9$, without a few galaxies that only have upper limits to $\mathrm{HCN}$ emission. This linear correlation continues to the Galactic massive cores, but a decline in $L_{I R}$ occurs at $10^{4.5} \mathrm{~L}_{\odot}$, below which the slope of the correlation becomes much steeper. When fit to Galactic cores with $L_{I R}>10^{4.5} \mathrm{~L}_{\odot}$, the linear least squares fit gives $\log \left(L_{I R}\right)=1.02( \pm 0.06) \times \log \left(L_{H C N}\right)+2.79( \pm 0.16)$. This relation agrees remarkably well with the relation for galaxies, as seen in fig. 1a. In fig. 1b, we fit simultaneously the data of galaxies from Gao \& Solomon (2004a) and Galactic cores with $L_{I R}>10^{4.5} \mathrm{~L}_{\odot}$. The result is $\log \left(L_{I R}\right)=1.01 \times \log \left(L_{H C N}\right)+2.83$, with a correlation coefficient of 0.99 . Understanding the physics behind this linear correlation will lead to a better understanding of star formation on galactic scales.

The linear $\log \left(L_{I R}\right)-\log \left(L_{H C N}\right)$ correlation and the turnoff can be seen more clearly from fig. 2, where the distance independent ratio $L_{I R} / L_{H C N}$ has been plotted versus $L_{I R}$ (top) and against $L_{m o l}$ (bottom). A constant mean value of $L_{I R} / L_{H C N}$ is seen over 8 orders of magnitude in $L_{I R}$, from galaxies to Galactic cores, as long as $L_{I R}>L($ min $)$, with $L(\min )$ being around $10^{4.5} \mathrm{~L}_{\odot}$. The corresponding cut-off value for $L_{H C N}$ is $L_{\text {unit }}=10^{1.5} \mathrm{~K}$ $\mathrm{km} \mathrm{s}^{-1} \mathrm{pc}^{2}$. For Galactic cores, $\left\langle L_{I R} / L_{H C N}\right\rangle=911 \pm 227$, with median 380. For galaxies, $\left\langle L_{I R} / L_{H C N}\right\rangle=950 \pm 76$, with median 855 . The uncertainties are the standard deviation of the means, which are remarkably similar, though the dispersion is much higher for Galactic cores. The median for dense cores is significantly smaller than its mean, which indicates that the mean is dominated by a few quite large values. The logarithmic mean and median are $2.64 \pm 0.53$ and $2.54 \pm 0.56$ for dense cores with $L_{H C N}>L_{u n i t}$, and $2.91 \pm 0.24$ and $2.93 \pm 0.25$ for galaxies.

For comparison, we added CO data on Galactic cores (Mooney \& Solomon 1988), galaxies (Gao \& Solomon 2004a) and high-z molecular emission line galaxies (Solomon \& Vanden Bout 2005) in fig. 2. The ratio, $L_{I R} / L_{C O}$, changes by two orders of magnitude as $L_{C O}$ 
increases from Galactic cores to distant galaxies, confirming Gao \& Solomon's conclusion that $\mathrm{CO}$ is not as good a tracer of star-forming gas as is HCN, especially for very luminous starburst galaxies.

\section{Discussion}

The fact that $L_{I R} / L_{H C N}$ is similar, on average, for star-forming cores in the Galaxy, normal spirals, starbursts, and ULIRGs suggests the possibility of interpreting intense high-Z star formation in terms of nearby high mass star forming regions. Before we can exploit this possibility, we must understand some key points. Why does $L_{I R} / L_{H C N}$ rise steeply with $L_{H C N}$ and then remain constant for $L_{H C N}>L_{\text {unit }}$ ? And why is the ratio, $L_{I R} / L_{C O}$, NOT constant for starbursts?

As a first step, we seek a more physical basis for the relations. We have so far discussed $L_{H C N}$ as a measure of the mass of dense gas, but can we quantify this assumption? A roughly linear correlation between the mass of dense gas and bolometric luminosity has been found by our work on CS and dust emission (Shirley et al. 2003; Mueller et al. 2002). To see whether this applies also to $\mathrm{HCN} J=1 \rightarrow 0$, we have calculated the virial mass of the dense gas $(M($ dense $))$ using the most optically thin line $\left(\mathrm{C}^{34} \mathrm{~S} J=5 \rightarrow 4\right)$ to measure the linewidth and compared $M$ (dense) to $L_{H C N}$. Based on the 31 cores with available $\mathrm{C}^{34} \mathrm{~S} J=5 \rightarrow 4$ data, we obtained the correlation: $\log \left(M_{v i r}\left(R_{H C N 1-0}\right)\right)=(0.81 \pm 0.03) \times \log \left(L_{H C N 1-0}\right)+1.29( \pm 0.09)$. The correlation is roughly linear. The $M_{v i r}-L_{H C N 1-0}$ plot and details of the observations of $\mathrm{C}^{34} \mathrm{~S} J=5 \rightarrow 4$ will be presented by Wu et al. (2005, in preparation). Leaving out one peculiar source (G35.58-0.03), we get $\left\langle M\right.$ (dense) $\left./ L_{H C N}\right\rangle=11 \pm 2 \mathrm{M}_{\odot} / \mathrm{K} \mathrm{km} \mathrm{s}^{-1} \mathrm{pc}^{2}$, where the uncertainty is the standard deviation of the mean; the median value is $6 \mathrm{M}_{\odot} / \mathrm{K} \mathrm{km} \mathrm{s}^{-1}$ $\mathrm{pc}^{2}$, indicating that the mean is affected by some quite large values. The logarithmic mean is $7 \pm 2 \mathrm{M}_{\odot} / \mathrm{K} \mathrm{km} \mathrm{s}^{-1} \mathrm{pc}^{2}$. Some of the scatter in the ratio may be caused by distance uncertainties because the virial mass depends linearly on distance, while $L_{H C N} \propto D^{2}$.

Even after establishing that $L_{H C N}$ traces the mass of dense gas, it is not at all clear why $L_{I R} / L_{H C N}$ should be constant, since the luminosity of a cluster is typically dominated by its most massive members, and $L_{\star} \propto M_{\star}{ }^{\alpha}$, with $\alpha \approx 3$ to 4 . Indeed, below the cutoff of $10^{4.5} \mathrm{~L}_{\odot}, L_{I R}$ does rise rapidly with $M$ (dense). What causes the transition to a constant value?

To solve this puzzle, we propose the existence of a basic unit of cluster formation. For $M$ (dense) less than the mass of this unit, $L_{I R} / M$ (dense) rises rapidly with $M($ dense), as higher mass stars can form. For $M$ (dense) greater than the mass of this unit, the IMF is 
reasonably sampled and further increases in mass produce more units, but no further change in $L_{I R} / M$ (dense). If we suppose that larger scale cluster formation is built up by adding more and more such units, then the linear correlation between the total $L_{I R}$ and $M$ (dense) is a natural result. In that case, the only difference between star formation on different scales and in different environments-big clusters, normal galaxies, massive ULIRGs-is just how many such cores they contain. This is of course somewhat of a simplification because the cores have a range of properties. The fact that the mean is roughly twice the median for both $L_{I R} / L_{H C N}$ and $M$ (dense) $/ L_{H C N}$ for Galactic cores suggests that the most extreme cases may dominate when averaged over a whole galaxy. Since most of our cores contain compact $\mathrm{H} \amalg$ or $\mathrm{UCH} \amalg$ regions, which trace the most massive stars, it is interesting to compare to the luminosity function of these regions. A study of Galactic UCH $\amalg$ regions did find a peak luminosity $\left(\sim 10^{5} \mathrm{~L}_{\odot}\right)$ that is close to that of a basic unit (Cassassus et al. 2000). The detailed discussion of this model will be presented in a separate paper (Wu et al. 2005, in preparation).

Another question is why does $L_{I R} / L_{C O}$ increase as we move from Galactic Cores or normal spiral galaxies to starburst galaxies. In Galactic clouds, $\mathrm{CO}$ can be used as a tracer of the overall mass of molecular clouds, even though it is optically thick and thermalized. However, CO does not specifically trace the mass of dense cores. For that, dust continuum emission, or molecules, like HCN, that are only excited at higher densities are required. In the Galaxy, these dense cores occupy a small fraction of the mass of a cloud (typically a few percent). If the fraction of dense gas in the overall cloud stays roughly constant, the relation between $L_{I R}$ and $L_{C O}$ can stay linear, but this relation is secondary to that between $L_{I R}$ and $M$ (dense). For starburst galaxies, the fraction of the molecular gas concentrated in dense cores increases, causing (Gao \& Solomon 2004a) the secondary relation between $L_{I R}$ and $L_{C O}$ to become non-linear.

These considerations lead us to offer some new versions of "Schmidt Laws":

$$
\begin{gathered}
\dot{M}_{\star}\left(\mathrm{M}_{\odot} y r^{-1}\right) \sim 1.4 \times 10^{-7} L_{H C N}\left(K \mathrm{~km} \mathrm{~s}^{-1} p c^{2}\right) \\
\dot{M}_{\star}\left(\mathrm{M}_{\odot} y r^{-1}\right) \sim 1.2 \times 10^{-8} M(\text { dense })\left(\mathrm{M}_{\odot}\right)
\end{gathered}
$$

where $\dot{M}_{\star}$ is the star formation rate, and we have assumed that $\dot{M}_{\star}\left(\mathrm{M}_{\odot} y r^{-1}\right)=2.0 \times 10^{-10} L_{I R}\left(\mathrm{~L}_{\odot}\right)$ (see Gao \& Solomon (2004b)) and we use the fit to both dense cores and galaxies. The coefficients $\left(1.4 \times 10^{-7}\right.$ and $\left.1.2 \times 10^{-8}\right)$ are very similar to, but slightly less than, those values $\left(1.8 \times 10^{-7}\right.$ and $\left.1.8 \times 10^{-8}\right)$ given by Gao \& Solomon $(2004 \mathrm{~b})$, based only on the galaxies.

Given these simpler, linear relations, how do we understand the usual star formation law (Kennicutt 1998) relations, with a steeper dependence $\left(\Sigma_{S F R} \propto \Sigma_{\text {gas }}^{1.4}\right)$ of $\dot{M}_{\star}$ on gas mass? We suggest that the steeper dependence reflects the dependence of $M$ (dense) on the surface 
density, or total mass, of gas. All the tracers of gas used by Kennicutt trace lower density components, not the actual gas that is directly involved in star formation. Once it is clear that it is the dense gas mass that indicates the star formation rate, it becomes clear why the total surface density of gas may not be a clean star formation indicator. For example, there is no evidence that HI emission in galaxies correlates at all with the star formation rate deduced form the far-infrared luminosity, so its contribution to the surface density may have no effect on the star formation rate. Attempts to provide a theoretical framework (e.g., Li et al. 2005, Krumholz \& McKee 2005) for the Kennicutt relations should also be able to explain the new relations.

It will be interesting to see how far these ideas can be extended. One future project is to look at $\mathrm{HCN}$ in more nearby galaxies, especially in individual regions forming superstar clusters, which may be the building blocks of star formation in starburst regions like ULIRGs and LIRGs (Ho 1997). ALMA will allow detailed study of HCN in other galaxies, including the $J=3 \rightarrow 2$ transition. Another is to explore how far toward higher $\mathrm{z}$ these relations can be pushed, and to understand how the relations depend on metallicity and chemistry. Theoretical work by Lintott et al. (2005) suggest that HCN may trace very early star formation, depending on the nucleosynthetic yield of the earliest stars.

We are grateful to Mark Heyer and other FCRAO staff for assistance with the observations. We thank the referee for helpful comments. We thank Dan Jaffe, John Scalo, Shardha Jogee, John Kormendy, Michael Dopita, and Luis Ho for helpful discussions. This work was supported by NSF Grant AST-0307250 to the University of Texas at Austin and by the State of Texas. YG acknowledges support of the NSF of China grants 10425313 \& 10333060. 


\section{REFERENCES}

Carilli, C. L., Solomon., P. M., Vanden Bout, P. A., Walter, F., Beelen, A., and Cox, P., Bertoldi, F., Menten, K. M., Isaak, K. G., Chandler, C. J., \& Omont, A. 2005, ApJ, 618,586

Carpenter, J. M. 2000, AJ, 120, 3139

Casassus, S., Bronfman, L., May, J., \& Nyman, L. -A. 2000, A\&A, 358, 514

Evans, N. J. II. 1991, in Frontiers of Stellar Evolution, ed. D. L. Lambert, (San Francisco: Astro. Soc. Pacific), 45

Gao, Y., \& Solomon, P. M., 2004a, ApJ, 606, 271

Gao, Y., \& Solomon, P. M., 2004b, ApJS, 152, 63

Greve, T. R., Bertoldi, F., Smail, Ian, Neri, R., Chapman, S. C., Blain, A. W., Ivison, R. J., Genzel, R., Omont, A., Cox, P., Tacconi, L., Kneib, J. -P. 2005, MNRAS, 359, 1165.

Ho, L. C. 1997, in Rev. Mexicana Astron. Astrofis. Conf., Vol. 6, 1st Guillermo Haro Conference on Astrophysics: Starburst Activity in Galaxies, 5

Isaak, K. G., Priddey, R. S., Mcmahon, R. G., Omont, A., Peroux, C., Sharp, R. G., and Withington, S. 2002, MNRAS, 329, 149

Kennicutt,R. C. 1997, in The Interstellar Medium in Galaxies, ed. J. M. van der Hulst (Dordrecht: Kluwer), 171

Kennicutt,R. C. 1998, ApJ, 498, 541

Kewley, L. J., Geller, M. J., Jansen R. A. \& Dopita, M. A. 2002, AJ, 124, 3135

Krumholz, M. R. \& McKee, C. F. 2005, ApJ, 630, 250

Lada, C. J. \& Lada, E. A. 2003, Ann. Rev. Ast. \& Astrophys., 41, 57

Li, Y., Mac Low, M.-M., \& Klessen, R. S. 2005, ApJ, 626, 823

Lintott, C. J., Viti, S., Williams, D. A., Rawlings, J. M. C., Ferreras, I. 2005, MNRAS, 360, 1527

Mead, K. N., Kutner, M. L. and Evans, N. J. II. 1990, ApJ, 354, 492

Mooney, T. J., \& Solomon, P. M. 1988, ApJ, 334, 51 
Mueller, K. E., Shirley, Y. L., Evans, N. J. II, \& Jacobson, H. R. 2002, ApJS, 143, 469

Plume, R., Jaffe, D. T., Evans, N. J. II. 1992, ApJS, 78, 505

Plume, R., Jaffe, D. T., Evans, N. J. II, Martin-Pintado, J., \& Gomez-Gonzalez, J. 1997, ApJ, 476, 730

Reuland, M., Röttgering, H., and van Breugel, W. 2003, New Astronomy Reviews, 47, 303

Sanders, D. B., \& Mirabel, I. F. 1996, Ann. Rev. Ast. \& Astrophys., 34, 749

Shirley, Y. L., Evans, N. J. II, Mueller, K. E., Knez, C., Jaffe, D. T. 2003, ApJS, 149, 375.

Solomon, P. M., Downes, D., Radford, S. J. E., Barrett, J. W. 1997, ApJ, 478, 144

Solomon., P. M., Vanden Bout, P. A.,Carilli, C. L., \& Guelin, M. 2003, Nature, 356, 318

Solomon., P. M. \& Vanden Bout, P. A. 2005, Ann. Rev. Ast. \& Astrophys. In press.

Schmidt, M. 1959, ApJ, 129, 243

Vanden Bout, P. A., Solomon., P. M., \& Maddalena, R. J. 2004, ApJ, 614, 97

Wu, J., \& Evans, N. J. II, 2003, ApJ, 592, 79

Wu, Y., Wei, Y., Zhao, M., Shi, Y., Yu, W., Qin, S., and Huang, M. 2004, A\&A, 426, 503

Zhang, Q., Hunter, T. R., Brand, J., Sridharan, T. K., Cesaroni, R., Molinari, S., Wang, J., and Kramer, M. 2005. AJ, in press 

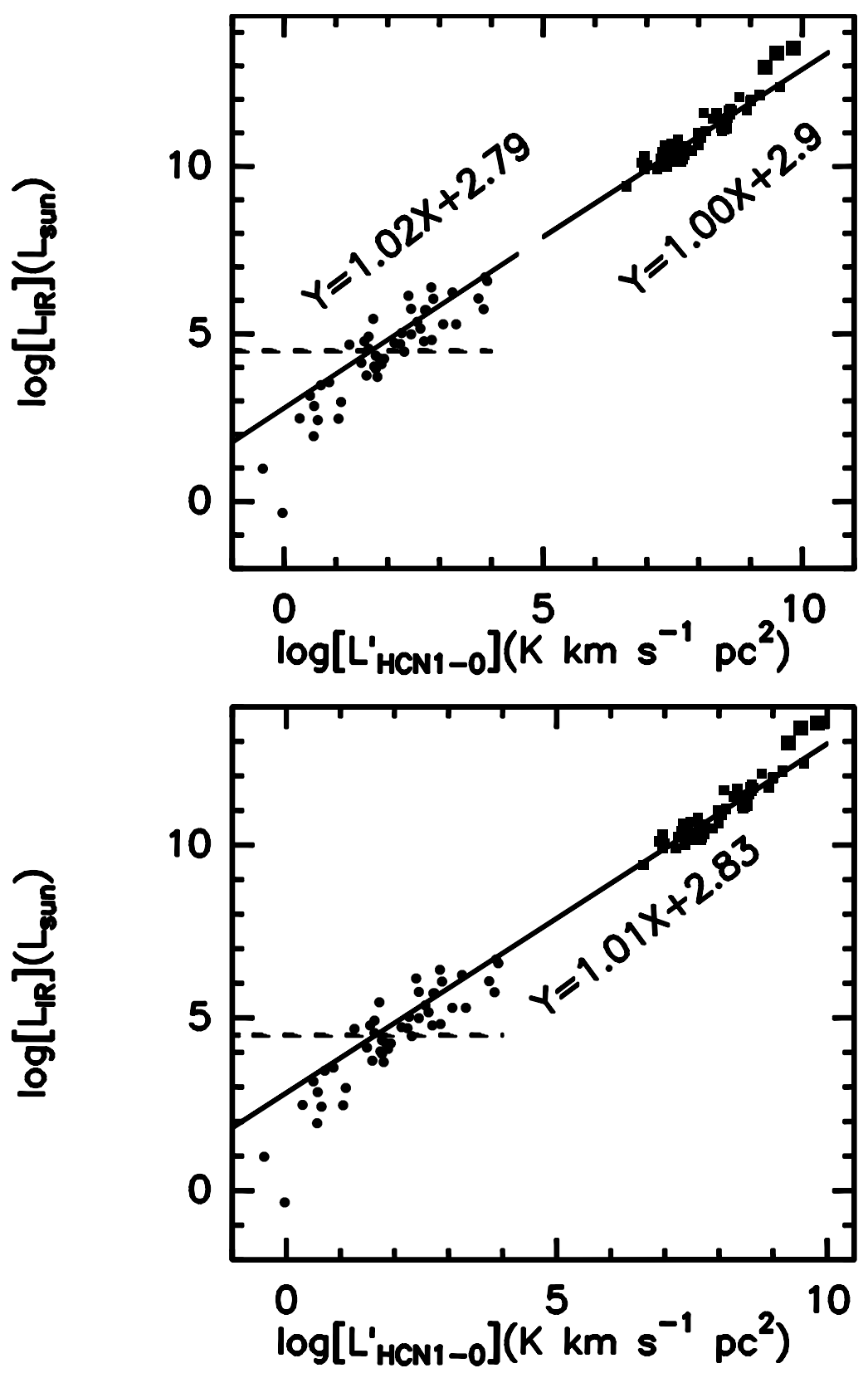

Fig. 1.- $\log \mathrm{L}_{I R}-\log \mathrm{L}_{H C N 1-0}^{\prime}$ correlation for Galactic and extragalactic sources. Fig.1a (above) indicates the linear least squares fit for Galactic cores (with $L_{I R}>10^{4.5} \mathrm{~L}_{\odot}$, points above the dashed line) and for galaxies, separately. Fig. 1b (down) shows a overall fit for both parts. The three isolated solid squares are high z HCN 1-0 points from Solomon et al. (2003), Vanden Bout et al. (2004) and Carilli et al. (2005); they are not included in the fit because the sources are QSOs and the contribution from the AGN to $L_{I R}$ is not yet clear. 

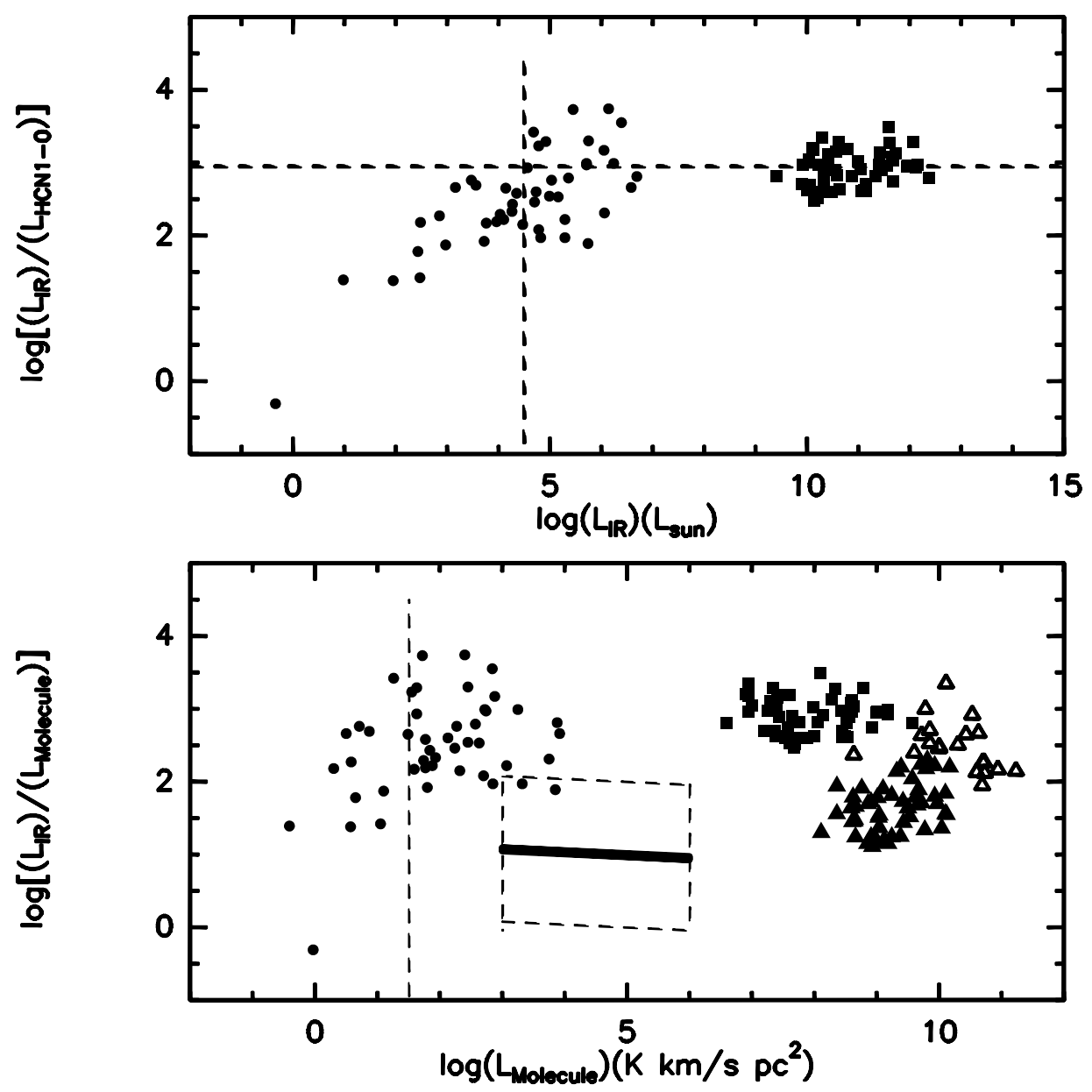

Fig. 2.- Above: Correlation between $\mathrm{L}_{I R}$ and $\mathrm{L}_{I R} / \mathrm{L}_{H C N 1-0}$ for galaxies (solid squares) and Galactic star forming cores (filled circles). $\mathrm{L}_{I R} / \mathrm{L}_{H C N 1-0}$ is constant for a large range of $\mathrm{L}_{I R}$ utill a turn off around $\mathrm{L}_{I R}=10^{4.5} \mathrm{~L}_{\odot}$. Below: $\mathrm{L}_{I R}$ per unit of molecular gas vs. molecular line luminosity. The star formation rate per amount of CO gas changes a lot from Galactic GMCs (the heavy line, with a dashed line boundary to indicate the variation), to galaxies (filled triangles) and high-z CO galaxies (hollow triangles). 